\title{
Tissue Inflammation Impairs Tissue-Level Perfusion and Promotes Left Ventricular Remodeling in Patients With Acute Myocardial Infarction
}

\author{
Yuya Nishida, MD*, Hiroshi Ito, MD*, Katsuomi Iwakura, MD*, \\ Kouji Tanaka, MD*, Shigeo Kawano, MD*, Atsunori Okamura, MD*, \\ Yoshinori Maekawa, MD*, Kouichi Inoue, MD*, Masatsugu Hori, MD** \\ and Kenshi Fujii MD* \\ *Division of Cardiology, Sakurabashi Watanabe Hospital \\ **Department of Internal Medicine and Therapeutics, Osaka University Post Graduate School, \\ Osaka, Japan
}

\begin{abstract}
Background. C-reactive protein (CRP) level and monocytosis are associated with left ventricular (LV) remodeling in patients with AMI.

Methods. One hundred twenty-nine consecutive patients with the first acute myocardial infarction (AMI) underwent myocardial contrast echocardiography (MCE) 2 weeks after successful reperfusion.

Results. LV end-diastolic volume index (LVEDVI) at pre-discharge was significantly higher in the no-reflow group than the reflow group (64 \pm 17 vs. $58 \pm 11 \mathrm{~mL} / \mathrm{m}^{2}, \mathrm{p}<0.01$ ). The no-reflow group had a higher CRP level and peak monocyte counts than the reflow group $(9.5 \pm 5.2$ vs. $5.8 \pm 3.5 \mathrm{mg} / \mathrm{dl}, \mathrm{p}<0.0001$; and $1026 \pm 400 \mathrm{vs} .824 \pm 278 / \mu \mathrm{l}, \mathrm{p}<0.001$, respectively). Peak CRP (relative risk [RR] 1.21, 95\% confidence interval [Cl] 1.01-1.45, $\mathrm{p}<0.05$ ) and peak monocyte counts (RR 1.003, 95\% Cl 1.001-1.006, p<0.01) were independent determinants of the no-reflow phenomenon.

Conclusion. Microvascular dysfunction following tissue inflammation may play an important role in the LV remodeling after AMI.

(J Echocardiogr 2005; 3: 83-90)
\end{abstract}

Key words: No-reflow, Inflammation, Monocyte, Myocardial infarction, Microcirculation

\section{Introduction}

Increasing evidences implicate the inflammation response after acute myocardial infarction (AMI) to be a factor related to patient mortality. C-reactive protein (CRP) dramatically increases after AMI [1], and its peak value is correlated to the mortality after AMI [2-3]. Elevated CRP level after AMI is also related to left ventricular aneurysm [4-5] and cardiac rupture [4]. Cytokine such as interleukin-6 also increased after AMI [6], and their level is correlated with mortality.

Received May 6, 2005; revision received July 11, 2005; accepted July 14, 2005

Address for correspondence: Hiroshi Ito, MD

Division of Cardiology, Sakurabashi Watanabe Hospital,

2-4-32 Umeda, Kita-ku, Osaka 530-0001, Japan.

Telephone: +81-6-6341-8651

Fax: +81-6-6341-0785.

E-mail: itomd@osk4.3web.ne.jp

(C) 2005 Japanese Society of Echocardiography
Another sign of tissue inflammation in AMI is an increase in the number of peripheral leukocytes. The higher white blood cell count is associated with an increase in the rate of mortality [7] and re-infarction [8] after AMI. A recent clinical study documented the observation that the peripheral monocyte count is correlated to left ventricular remodeling and cardiac events after AMI [9]. However, the mechanism by which the inflammatory response affects the ventricular remodeling after AMI is still not fully understood as yet.

The no-reflow phenomenon is another factor that is associated with poor functional and clinical outcomes after AMI [10-13]. Myocardial contrast echocardiography (MCE) demonstrated that the no-reflow phenomenon is observed in $25 \%$ to $30 \%$ of patients with AMI despite successful coronary recanalization [10, 14]. The no-reflow phenomenon may persist in subacute phase, and correlate with progression of microvascular dysfunction. Several mechanisms are postulated to 
explain the development of the no-reflow phenomenon [15-16], however, the contribution of tissue inflammation remains unknown. Ott et al. reported that monocyte Mac-1 expression and monocyte procoagulant activity increased after angioplasty along with the levels of interleukin-6 and CRP in patients with AMI [17]. To clarify whether this procoagulant inflammatory response of monocytes is related to the no-reflow phenomenon after AMI, we examined the parameters that reflect inflammatory responses in relation to the noreflow phenomenon in patients with AMI.

\section{Methods}

\section{Study population.}

One hundred eighty-six consecutive patients with the first AMI who were admitted to Sakurabashi Watanabe Hospital were enrolled in this study between June 2001 and August 2003. All patients underwent primary percutaneous coronary intervention (PCI, angioplasty and/or stent) for arteries exhibiting Thrombolysis in Myocardial Infarction (TIMI) flow grade 0 or 1 within 24 hours after symptom onset; subsequently, they underwent $\mathrm{MCE}$ examination. The AMI diagnosis was based on prolonged chest pain $\geq$ 30 minutes, ST segment elevation of $\geq 2 \mathrm{~mm}$ in at least two contiguous electrocardiography (ECG) leads, and greater than a three-fold increase in serum creatine kinase levels. Fifty-seven patients were excluded; 32 patients suffered cardiogenic shock; in 16 patients association of collagen disease was observed; and 9 patients showed malignancy or infectious disease. Therefore, the final study population consisted of 129 patients. The study protocol was approved by the hospital's Ethics Committee, and patients gave their written informed consent.

\section{Study protocol}

Immediately after admission, a 12-leads ECG was recorded and the blood glucose level was measured in each patient. All patients underwent two-dimensional echocardiography with a SONOS 5500 system (Philips Medical Systems). We performed coronary angioplasty using the appropriate balloon catheters. We repeated angioplasty or implanted a stent to reduce residual diameter stenosis to $<50 \%$. On admission to the hospital, venous blood samples were obtained every 24 hours for at least 5 days. Total and differential leukocyte counts were measured by an automated hematology analyzer (MAXM-Retic, BECKMAN COULTER, Tokyo, Japan). Serum creatine kinase (CK) activity and CRP levels were measured by latex photometric immunoassay (SYNCHRON LX20, BECKMAN COULTER, Tokyo, Japan) with the use of an autoanalyzer (RadiSys, RadiSys Corporation) to obtain the peak CK and CRP levels.

We performed MCE 2 weeks after the onset of AMI using SONOS5500 (Philips Medical Systems) with the S3 probe. We depicted apical 4- and 2-chamber views with harmonic power Doppler (HPD) mode [18-20], and ultrasound was transmitted at $1.8 \mathrm{MHz}$ and received at $3.6 \mathrm{MHz}$. The focus was placed at the mitral level, and the mechanical index and dynamic range were set to the maximum, 1.6 and $40 \mathrm{~dB}$, respectively. Overall gain was adjusted to minimize artifacts in the baseline study. A solution of Levovist ${ }^{\mathrm{TM}}$ (concentration of $300 \mathrm{mg} / \mathrm{mL}$, Schering AG) [19-21] was administrated as bolus $(3 \mathrm{~mL})$ at a rate of $0.5 \mathrm{~mL} / \mathrm{s}$ with a volumetric pump (PULSAR, Medrad). MCE images were recorded for at least 180 seconds after the contrast injection. End-systolic images were obtained every 4 heart beats, and they were stored on a magneto-optical disk. Imaging was performed with 2 triggers (multi-frame triggering mode) [22]. The first pulse was used for myocardial opacification ; the second pulse was used only for bubble destruction to assess the quality of images, and we reduced the motion artifact as much as possible by adjusting the triggering time.

\section{Analysis of MCE data}

Left ventricular myocardium was divided into 6 segments using both apical 4- and 2-chamber views according to the segmentation of the American Society of Echocardiography. We analyzed MCE images 100 seconds after injection to evaluate the perfusion defect. At each myocardial segment, we judged the presence of the perfusion defect. The perfusion defect was defined as the lack of staining of myocardial tissue at the end systole (Figure 1). If there were at least one segment that showed the perfusion defect, we defined it as the no-reflow group, and if no perfusion defect was detected, we evaluated as reflow group. The evaluation was not performed if any of the following features was present: absolutely no contrast enhancement, termed drop-out, and apparent motion artifacts. This judgment was taken by at least two well-trained sonographers.

\section{Statistical analysis}

Continuous data are expressed as the mean value \pm SD. Comparison between two groups was performed 

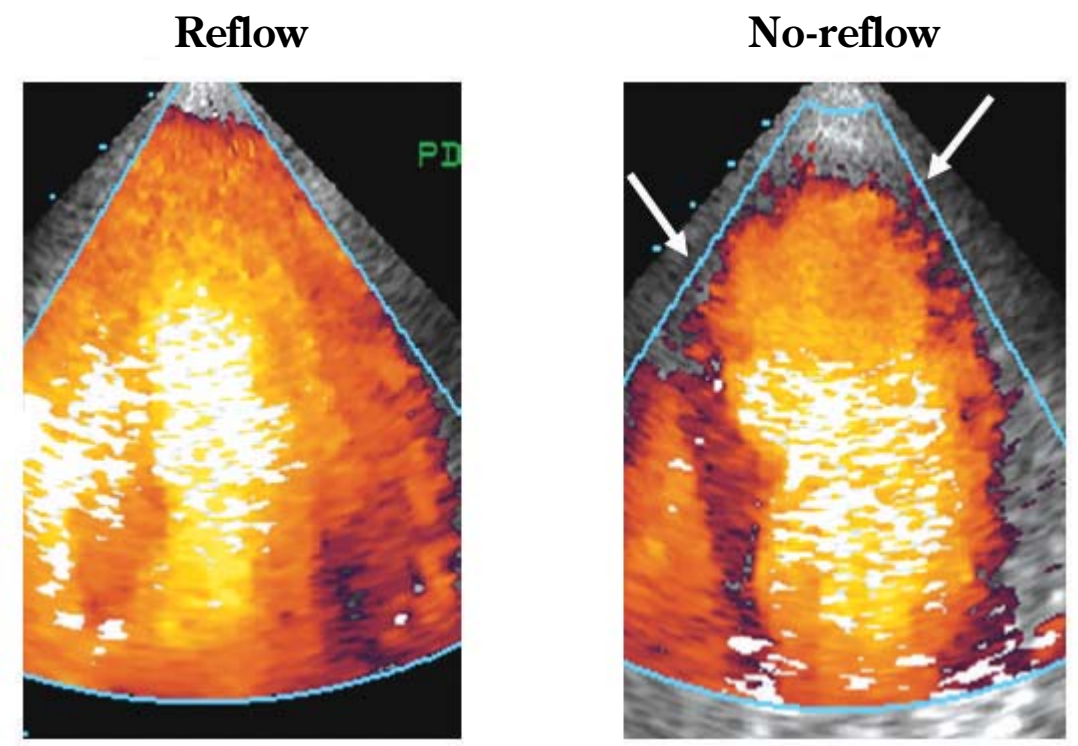

Fig. 1. Myocardial contrast echocardiographic (MCE) images in the patients with reperfused AMI.

In the image on the left, myocardial perfusion was well preserved in all myocardial segments. On the other hand, the image on the right shows perfusion defect within apical lesions, which is indicated between the arrows.

Table 1. Patient characteristics between two groups

\begin{tabular}{lccc}
\hline & $\begin{array}{c}\text { Reflow } \\
(\mathrm{n}=81)\end{array}$ & $\begin{array}{c}\text { No-reflow } \\
(\mathrm{n}=48)\end{array}$ & $\begin{array}{c}\mathrm{P} \\
\text { value }\end{array}$ \\
\hline Age & $60 \pm 10$ & $62 \pm 11$ & 0.33 \\
Male gender & $73(90)$ & $40(83)$ & 0.26 \\
Hypertension & $40(49)$ & $21(44)$ & 0.54 \\
Current smoking & $62(77)$ & $35(73)$ & 0.64 \\
Hyperlipidemia & $41(51)$ & $15(31)$ & 0.03 \\
Diabetes mellitus & $26(32)$ & $15(31)$ & 0.92 \\
Anterior MI & $20(25)$ & $37(77)$ & $<0.0001$ \\
Preinfarction angina & $42(52)$ & $26(54)$ & 0.8 \\
ACE inhibitors & $15(19)$ & $12(25)$ & 0.38 \\
Elapsed time & $5.1 \pm 4.3$ & $6.4 \pm 5.4$ & 0.14 \\
Stent use & $70(86)$ & $42(88)$ & 0.86 \\
\hline
\end{tabular}

Values are expressed as number(\%) or mean \pm SD unless otherwise noted. $\mathrm{ACE}=$ angiotensin-converting enzyme 

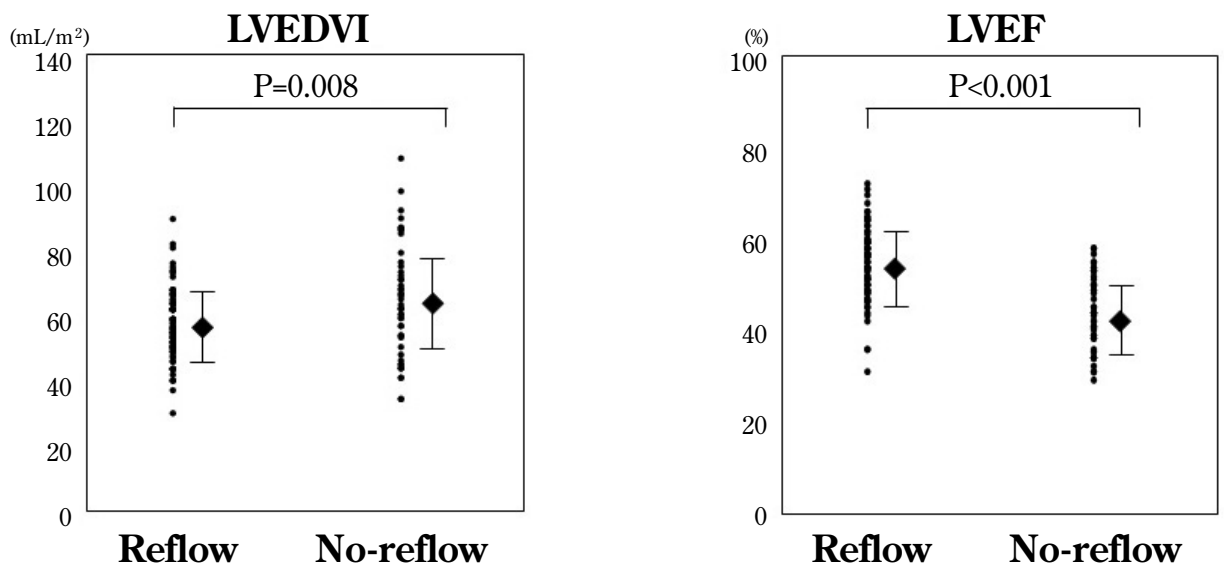

Fig. 2. The left ventricular end diastolic volume index (LVEDVI) and ejection fraction (EF) at the follow up study.

LVEDVI and EF were measured in the left ventriculogram performed after an average gap of 5 months after AMI onset. The group showing no-reflow on MCE had significantly higher LVEDVI (left) and lower EF (right) than those with good reflow. The bars demonstrate the mean \pm SD values.
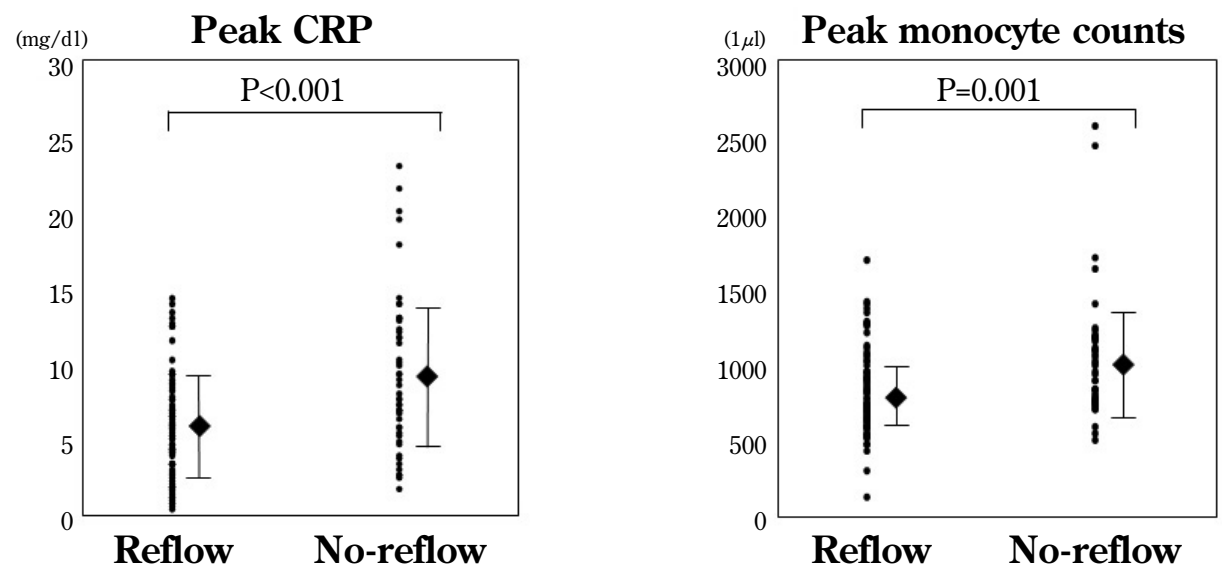

Fig. 3. Peak CRP levels and peak monocyte counts at the early stage of AMI. The no-reflow group had significantly higher peak CRP levels (left) and peak monocyte counts (right) than the good reflow group.

by using the unpaired $t$-test for continuous variables and by using the chi-square test for categorical variables. Multiple logistic regression analysis was used to assess the effect of various factors on the no-reflow phenomenon. Variables, including those with $\mathrm{p}<0.10$ obtained by univariate analysis, were further investigated by a multiple logistic regression test. $\mathrm{P}$ value < 0.05 was considered statistically significant. All statistical analyses were performed using Statview 5.0 software (SAS Institute Inc., Cary, North Carolina).

\section{Results}

\section{Patient characteristics}

Of the total number of patients who entered this study, 113 were men and 16 were women, with a mean age of $61 \pm 11$ (range 38 to 89 ). The average interval from the symptom onset to the arrival at hospital was 4.4 hours, and the interval to reperfusion was 5.8 hours. The patients were divided into two groups based on the MCE findings, the no-reflow group ( $\mathrm{n}=$ $48,37 \%)$ and the reflow group $(\mathrm{n}=81,63 \%)$. There 
were no significant differences in age, gender, symptom onset-reperfusion time, and use of stents between these two groups. Hyperlipidemia and anterior wall infarction were more frequently seen in the no-reflow group than in the reflow group (Table 1). Three weeks after the symptom onset the left ventricular end-diastolic volume index was higher in the no-reflow group than in the reflow group $\left(64 \pm 17 \mathrm{vs} .58 \pm 11 \mathrm{~mL} / \mathrm{m}^{2}, \mathrm{p}\right.$ $<0.01)$. In contrast, the left ventricular ejection fraction was lower in the no-reflow group than in the reflow group ( $45 \pm 9 \%$ vs. $55 \pm 8 \%, \mathrm{p}<0.001)$ (Figure 2).

\section{Factors related to the no-reflow phenomenon}

There was no difference in the interval from symptom onset to peak CRP between the two groups, and this interval was approximately 3 days from reperfusion. Peak CRP was higher in the no-reflow group than in the reflow group $(9.5 \pm 5.2$ vs. $5.8 \pm 3.5 \mathrm{mg} / \mathrm{dl}$, p $<0.0001$ ) (Figure 3). Although the peak number of leukocytes was comparable between the two groups

Table 2. Univariate analysis for no-reflow phenomenon

\begin{tabular}{lccc}
\hline & $\begin{array}{c}\text { Reflow } \\
(\mathrm{n}=81)\end{array}$ & $\begin{array}{c}\text { No-reflow } \\
(\mathrm{n}=48)\end{array}$ & $\begin{array}{c}\mathrm{P} \\
\text { value }\end{array}$ \\
\hline Peak CK & $2278 \pm 1450$ & $5255 \pm 2869$ & $<0.0001$ \\
Peak WBC & $11436 \pm 2849$ & $12238 \pm 3229$ & 0.14 \\
BG on admission & $140 \pm 31$ & $157 \pm 41$ & 0.013 \\
Peak monocyte counts & $824 \pm 278$ & $1026 \pm 400$ & 0.001 \\
Peak CRP & $5.8 \pm 3.5$ & $9.5 \pm 5.2$ & $<0.0001$ \\
ST re-elevation & $23(28)$ & $31(65)$ & $<0.0001$ \\
WMS on admission & $11.5 \pm 4.6$ & $17.6 \pm 6.7$ & $<0.0001$ \\
Killip>2 & $5(6)$ & $14(29)$ & 0.0004 \\
\hline
\end{tabular}

Values are expressed as number(\%) or mean \pm SD unless otherwise noted.

$\mathrm{BG}=$ blood glucose $\mathrm{WMS}=$ wall motion score

Table 3. Multivariate analysis for no-reflow phenomenon

\begin{tabular}{lcccc}
\hline & \multicolumn{3}{c}{ Relative } & P \\
& $\chi^{2}$ & risk & $95 \%$ CI & value \\
\hline Age & 12.1 & 1.08 & $1.01-1.16$ & 0.026 \\
Male gender & 1.02 & 0.41 & $0.07-2.31$ & 0.31 \\
Peak CK & 12.2 & 1.001 & $1.000-1.001$ & 0.0005 \\
Peak monocyte & 6.92 & 1.003 & $1.001-1.006$ & 0.009 \\
Reperfusion injury & 4.60 & 4.26 & $1.13-16.0$ & 0.032 \\
Peak CRP & 4.21 & 1.21 & $1.01-1.46$ & 0.040 \\
Anterior MI & 4.16 & 3.65 & $1.05-12.7$ & 0.042 \\
WMS on admission & 1.96 & 1.11 & $0.96-1.27$ & 0.16 \\
BG on admission & 0.38 & 1.005 & $0.99-1.02$ & 0.54 \\
Hyperlipidemia & 0.22 & 0.76 & $0.24-2.38$ & 0.64 \\
Killip $>2$ & 0.05 & 1.25 & $0.18-8.65$ & 0.82 \\
\hline
\end{tabular}

$\mathrm{CI}=$ confidence interval; $\mathrm{CK}=$ creatine kinase 
$(12238 \pm 3229$ vs. $11436 \pm 2849 / \mu 1, \mathrm{p}=0.14)$, peak monocyte counts were higher in the no-reflow group than in the reflow group ( $1026 \pm 400$ vs. $825 \pm 278 / \mu 1$, $\mathrm{p}=0.001$ ) (Figure 3 ). This interval was approximately 2 days from reperfusion. The ST re-elevation was more frequently observed immediately after coronary recanalization in the no-reflow group than in the reflow group $(31 / 48,65 \%$ vs. $23 / 81,28 \%, \mathrm{p}<0.0001$ ) (Table $2)$. However, presence or absence of ST re-elevation was not correlated with peak CRP levels $(p=0.21)$. In all cases, there were no subacute occlusion of culprit lesion in coronary angiography 3 weeks after AMI.

\section{The predictive factors of the no-reflow phenomenon}

In the univariate analysis, the factors related to the no-reflow phenomenon were as follows: anterior MI, peak CK, peak monocytes counts, peak CRP, Killip $>2$ on admission, blood glucose on admission, ST re-elevation, hyperlipidemia, and the wall motion score (WMS) on admission (Table 2). Multiple logistic regression analysis showed that anterior MI (relative risk [RR] $3.65,95 \%$ confidence interval $[\mathrm{CI}] 1.05$ to $12.7, \mathrm{p}=$ 0.042), peak CK (RR 1.001, 95\% CI 1.000 to $1.001, \mathrm{p}=$ 0.0005), peak CRP (RR 1.21, 95\% CI 1.01 to $1.46, \mathrm{p}=$ 0.040 ), the presence of ST re-elevation (RR 4.26, 95\% CI 1.13 to $16.0, \mathrm{p}=0.032$ ), and peak monocyte counts (RR $1.00395 \%$ CI 1.001 to $1.006, \mathrm{p}=0.009$ ) were independent predictive factors of the no-reflow phenomenon (Table 3).

\section{Discussion}

In the present study, we investigated the relationship between the inflammatory response and the noreflow phenomenon in patients with first reperfused AMI. Left ventricular end diastolic volume index was greater and the left ventricular ejection fraction was lower in the no-reflow group than in the reflow group 3 weeks after AMI. The no-reflow group demonstrated two weeks after AMI showed higher monocyte counts and CRP levels than the reflow group. Multivariate analysis revealed that peak monocyte count and peak CRP level were independent factors that are associated with the no-reflow phenomenon. Our results suggest that a possible role of tissue inflammation on the coronary microcirculation and incomplete tissue perfusion is associated with the deterioration of functional outcomes and left ventricular remodeling 3 weeks after AMI.

\section{Tissue inflammation and the no-reflow phenomenon}

Leukocytes increase in the early stage of AMI, and this is used as a parameter to diagnose AMI. Neutrophil plugging in post-ischemic capillary networks is a cause of the no-reflow phenomenon, and this process is accelerated by coronary reperfusion. Our result, however, demonstrated that leukocyte count in itself is not indicative of the no-reflow phenomenon. Recently, Maekawa et al. reported that peripheral monocytes increase in the patients with AMI. The higher peak monocyte count was associated with the worst short and long-term outcomes as well as with the left ventricular remodeling after AMI [9]. In the region of severe ischemic damage, monocytes adhere to the endothelial wall, migrate through it into the infarcted tissue, and finally transform themselves to macrophages. Previous experimental study demonstrated that an increase in peripheral monocytes precedes an increase in macrophages in the infarcted tissue [23]. The macrophages initiate a potent inflammation process in tissue. They release several cytokines, including IL-6, IL-1, and TNF- $\alpha$, to accelerate tissue inflammation and edema and, thus, injure capillary networks. These cytokines also activate neutrophils and endothelial cells, promote the adherence of neutrophils to the endothelial cells resulting in their transendothelial migration and tissue injury, and finally, further worsen tissue perfusion [24].

Post-inflammatory cytokines and acute phase reactants, such as CRP, also increase in the acute stage of AMI. These cytokines induce liver cells to produce CRP. Ikeda et al. reported a close correlation between the serum IL-6 levels and CRP levels in AMI patients [6]. As a consequence of AMI, severe tissue inflammation occurs with more monocytes infiltrating into the infarcted tissue to produce the extensive capillary obstruction and myocardial necrosis. This process might be detected with the higher CRP level in this study.

In previous studies, we have reported the independent predictors related to the no-reflow phenomenon that was documented immediately after coronary reperfusion. They include the initial WMS as the size of risk area, the development of $\mathrm{Q}$ wave on admission, patent infarct-related artery, preconditioning angina, and acute-stage hyperglycemia [25]. In this study, we evaluated tissue perfusion in the convalescence stage in order to reduce the impact of microemboli on microcirculation as much as possible and to evaluate total capillary damage that is caused by ischemia as well as by reperfusion injury. The determinant factors that are 
associated with the no-reflow phenomenon are different from those of the acute phase. For example, high blood glucose levels positively correlated with the noreflow phenomenon; however, it was no longer an independent factor of the no-reflow phenomenon in this study.

\section{Poor myocardial perfusion and $L V$ remodeling}

Progressive LV remodeling is occasionally observed in patients surviving AMI, and it delays their prognosis [26-29]. Several factors related to LV remodeling are postulated, such as infarct size, anterior location, transmurality of infarction, loading condition, and patency of the infarct-related artery [26-28]. Recently, the importance of microvascular obstruction has been stressed. $\mathrm{Wu}$ and colleagues have reported that microvascular obstruction assessed by MRI 10 days after the onset of AMI predicts LV remodeling and poor prognosis [30]. In the ischemia/reperfusion rat model, the no-reflow phenomenon persists for one month after reperfusion, and it predicts worse scar thinning and infarct expansion [31]. We studied coronary microvascular function from the coronary blood flow velocity pattern, recorded by transthoracic Doppler, in the patients with reperfused anterior MI. Prolonged microvascular dysfunction, as assessed from short diastolic deceleration time, is associated with $\mathrm{LV}$ remodeling.

Besides salvaging the jeopardized myocardium in the epicardial layer, better myocardial perfusion accelerates the healing process of infarct tissue. Tissue perfusion accelerates the washout of infarct debris, the migration of leukocytes, and augments the healing process of the infarct tissue that is resistant to mechanical stress. In contrast, impairment of tissue perfusion for a long period due to capillary obstruction delays the healing process of the infarct tissue, resulting in infarct expansion and subsequent LV remodeling.

\section{Study limitations}

This is a retrospective study and selection bias might be included. However, we enrolled consecutive AMI patients who survived at least 2 weeks after the symptom onset and underwent MCE. Second, the serum level of monocyte-related cytokines, such as IL6, M-CSF, and MCP-1, might be required to study the function of monocytes. In the clinical study, there was no evidence to demonstrate that an increase in peripheral monocytes is associated with tissue macrophage in the infarct region, although experimental studies have documented it.

\section{Conclusions}

Tissue inflammation is an important factor that impairs tissue-level perfusion. Microvascular dysfunction may play an important role in the development of $\mathrm{LV}$ remodeling after reperfused AMI.

\section{References}

1. Kushner I, Broder ML, Karp D. Control of the acute phase response. Serum C-reactive protein kinetics after acute myocardial infarction. J Clin Invest 1978; 61(2): 235-42.

2. Pietila KO, Harmoinen AP, Jokiniitty J, Pasternack AI. Serum C-reactive protein concentration in acute myocardial infarction and its relationship to mortality during 24 months of follow-up in patients under thrombolytic treatment. Eur Heart J 1996; 17(9): 1345-9.

3. Kinjo K, Sato H, Osaka Acute Coronary Insufficiency Study (OACIS) Group. Impact of high-sensitivity C-reactive protein on predicting long-term mortality of acute myocardial infarction. Am J Cardiol 2003; 91(8): 931-5.

4. Anzai T, Yoshikawa T, Shiraki H, et al. C-reactive protein as a predictor of infarct expansion and cardiac rupture after a first Q-wave acute myocardial infarction. Circulation 1997; 96(3): 778-84.

5. Takahashi T, Anzai T, Yoshikawa T, et al. Serum C-reactive protein elevation in left ventricular remodeling after acute myocardial infarction-role of neurohormones and cytokines. Int J Cardiol 2003; 88(2-3): 257-65.

6. Ikeda U, Ohkawa F, Seino Y, et al. Serum interleukin 6 levels become elevated in acute myocardial infarction. J Mol Cell Cardiol 1992; 24(6): 579-84.

7. Barron HV, Harr SD, Radford MJ, et al. The association between white blood cell count and acute myocardial infarction mortality in patients $>$ or $=65$ years of age: findings from the cooperative cardiovascular project. J Am Coll Cardiol 2001; 38(6): 1654-61.

8. Hajj-Ali R, Zareba W, Ezzeddine R, Moss AJ. Relation of the leukocyte count to recurrent cardiac events in stable patients after acute myocardial infarction. Am J Cardiol 2001; 88(11): 1221-4.

9. Maekawa Y, Anzai T, Yoshikawa T, et 1. Prognostic significance of peripheral monocytosis after reperfused acute myocardial infarction: a possible role for left ventricular remodeling. J Am Coll Cardiol 2002; 39(2): 241-6.

10. Ito H, Tomooka T, Sakai N, et al. Lack of myocardial perfusion immediately after successful thrombolysis. A predictor of poor recovery of left ventricular function in anterior myocardial infarction. Circulation 1992; 85(5): 1699705.

11. Ito H, Maruyama A, Iwakura $\mathrm{K}$, et al. Clinical implications of the 'no reflow' phenomenon. A predictor of com- 
plications and left ventricular remodeling in reperfused anterior wall myocardial infarction. Circulation 1996; 93(2): 223-8.

12. Reffelmann T, Kloner RA. The "no-reflow" phenomenon: basic science and clinical correlates. Heart 2002; 87(2): 162-8. Review.

13. Rezkalla SH, Kloner RA. No-reflow phenomenon. Circulation 2000; 105(5): 656-62. Review.

14. Porter TR, Li S, Oster R, Deligonul U. The clinical implications of no reflow demonstrated with intravenous perfluorocarbon containing microbubbles following restoration of Thrombolysis In Myocardial Infarction (TIMI) 3 flow in patients with acute myocardial infarction. Am J Cardiol 1998; 82(10): 1173-7.

15. Engler RL, Schmid-Schonbein GW, Pavelec RS. Leukocyte capillary plugging in myocardial ischemia and reperfusion in the dog. Am J Pathol 1983; 111(1): 98-111.

16. Sheridan FM, Cole PG, Ramage D. Leukocyte adhesion to the coronary microvasculature during ischemia and reperfusion in an in vivo canine model. Circulation 1996; 93(10): 1784-7.

17. Ott I, Neumann FJ, Kenngott S, Gawaz M, Schomig A. Procoagulant inflammatory responses of monocytes after direct balloon angioplasty in acute myocardial infarction. Am J Cardiol 1998; 82 (8): 938-42.

18. Senior R, Kaul S, Soman P, Lahiri A. Power doppler harmonic imaging: a feasibility study of a new technique for the assessment of myocardial perfusion. Am Heart J 2000; 139 (2 Pt 1): 245-51.

19. Agati L, Funaro S, Bilotta F. Assessment of no-reflow phenomenon after acute myocardial infarction with harmonic angiography and intravenous pump infusion with Levovist: comparison with intracoronary contrast injection. J Am Soc Echocardiogr 2000; 14(8): 773-81.

20. Yukiiri K, Ohmori K, Kondo I, et al. Assessment of myocardial viability in prior myocardial infarction by intravenous bolus microbubble injection: a new time domain index to estimate regional relative myocardial blood volume. J Am Soc Echocardiogr 2002; 15(8): 798806.

21. Wei K, Jayaweera AR, Firoozan S, et al. Quantification of myocardial blood flow with ultrasound-induced destruction of microbubbles administered as a constant venous infusion. Circulation 1998; 97(5): 473-83.
22. Yano A, Ito H, Iwakura K, et al. Myocardial contrast echocardiography with a new calibration method can estimate myocardial viability in patients with myocardial infarction. J Am Coll Cardiol 2004; 43(10): 1799-806.

23. Meisel SR, Pauzner H, Shechter M, Zeidan Z, David D. Peripheral monocytosis following acute myocardial infarction: incidence and its possible role as a bedside marker of the extent of cardiac injury. Cardiology 1998; 90(1): 52-7.

24. Iliceto S, Galiuto L, Marchese A, Colonna P, Oliva S, Rizzon P. Functional role of microvascular integrity in patients with infarct-related artery patency after acute myocardial infarction. Eur Heart J 1997; 18(4): 618-24.

25. Iwakura K, Ito $\mathrm{H}$, Ikushima $\mathrm{M}$, et al. Association between hyperglycemia and the no-reflow phenomenon in patients with acute myocardial infarction. J Am Coll Cardiol 2003; 41(1): $1-7$.

26. Ito H, Okamura A, Iwakura K, et al. Myocardial perfusion patterns related to thrombolysis in myocardial infarction perfusion grades after coronary angioplasty in patients with acute anterior wall myocardial infarction. Circulation 1996; 93(11): 1993-9.

27. Pfeffer MA, Braunwald E. Ventricular remodeling after myocardial infarction. Experimental observations and clinical implications. Circulation 1990; 81(4): 1161-72. Review.

28. Erlebacher JA, Weiss JL, Weisfeldt ML, Bulkley BH. Early dilation of the infarcted segment in acute transmural myocardial infarction: role of infarct expansion in acute left ventricular enlargement. J Am Coll Cardiol 1984; 4(2): 201-8.

29. Mallory GK, White PD, Salcedo-Salgar J. The speed of healing of myocardial infarction: a study of the pathologic anatomy in 72 cases. Am Heart J 1939; 18: 647-71

30. Wu KC, Zerhouni EA, Judd RM, Lugo-Olivieri $\mathrm{CH}$, Barouch LA, Schulman SP, Blumenthal RS, Lima JA. Prognostic significance of microvascular obstruction by magnetic resonance imaging in patients with acute myocardial infarction. Circulation 1998; 97(19): 765-72.

31. Reffelmann T, Hale SL, Dow JS, Kloner RA. No-reflow phenomenon persists long-term after ischemia/reperfusion in the rat and predicts infarct expansion. Circulation 2003; 108(23): 2911-7. 